\title{
METHYLPHENIDATE RESPONSE PREDICTION IN ADHD
}

Predictors of response to methylphenidate (MPH) among 47 children with ADHD and mental retardation (MR) were studied at the University of Pittsburgh School of Medicine, and the Western Psychiatric Institute and Clinic, Pittsburgh, PA. A double-blind, placebo-controlled evaluation of two doses of MPH $(0.3$ and $0.6 \mathrm{mg} / \mathrm{kg})$, using Conners Scales, with data collected on weekday and Saturday laboratory classrooms, showed that: 1) higher parent ratings of impulsivity and activity level at baseline were associated with greater gains in weekday classroom dependent measures; 2) higher weekday teacher measures of activity level, impulsivity, inattention, and conduct problems at baseline were related to improvement on Saturday laboratory classroom dependent measures; and 3) male Caucasians of higher socioeconomic status were more likely to show clinical improvements than other subjects. Race and conduct problems had predictive utility specific to children with MR. (Handen BL et al. Prediction of response to methylphenidate among children with ADHD and mental retardation. I Am Acad Child Adolesc Psychiatry October 1994;33:1185-1193). (Reprints: Dr Handen, John Merck Outpatient Program, Western Psychiatric Institute and Clinic, 3811 O'Hara St, Pittsburgh, PA 15213).

COMMENT. The higher the level of hyperactivity and associated inattention in children with ADHD and MR, the greater the beneficial response to methylphenidate. Similar findings have been reported previously in a study of 30 children of normal IQ with ADDH, using actometer measurements of motor activity and a neuropsychological test battery, and also in animals with hyperactivity induced by prefrontal cortical lesions. (Millichap JG. Ann N Y Acad Sci 1973;205:321; Ped Neur Briefs Oct 1987; June 1991).

Children with ADD without hyperactivity or moderate levels of abnormality on Conners Scales are less likely to benefit from stimulant medication than those with ADD and $\mathrm{H}$. Baseline measures of behavior by Conners Scales in ADDH children with MR were more predictive of medication response than the measures obtained in the laboratory classroom.

\section{METHYLPHENIDATE IN ADDH AND EPILEPSY}

The safety of methylphenidate (MPH), $0.3 \mathrm{mg} / \mathrm{kg}$, in 9 boys and 8 girls, ages 6 to 16 years, with ADHD and epilepsy, studied in Jerusalem, Israel, was reported at the Annual Meeting of the Child Neurology Society, Oct 2-8, 1994, in San Francisco, CA. Of 17 patients treated with MPH for 1-month, following a placebo period of 1 -month, 15 children who were seizure-free had no recurrence of seizures, while 2 with 1 to 2 seizures weekly before MPH had a moderate exacerbation of epilepsy. The EEGs showed no "major" changes. AED levels (CBZ and VA) were therapeutic during placebo and MPH periods. ADHD symptoms were benefited by MPH in 12 patients. (Gross-Tsur V et al. Methylphenidate for children with epilepsy and attention deficit hyperactivity disorder. Ann Neurol Sept 1994;36:501 [abstr]).

COMMENT. The authors recommend caution in the use of methylphenidate in ADHD children with an active seizure disorder. The PDR states that: "In the presence of seizures, the drug (MPH) should be discontinued." Based on the present and previous reports there appears to be some justification for trials of MPH in selected patients with ADDH 
and epilepsy whose seizures are controlled with antiepileptic drugs. Pemoline (Cylert) is generally considered to have less tendency to lower seizure threshold than MPII. Some recommend an EEG in all ADDH patients considered for stimulant medications; those with a history of seizures and/or epileptiform discharges in the EEG should receive concomitant AED therapy. Children with ADDH have a $7 \%$ incidence of epileptiform EEGs. (Ped Neur Briefs Oct 1989; Progress in Pediatric

Neurology I, 1991, p 190).

\section{COGNITIVE DEFICITS AND DEPRESSIVE SYMPTOMS}

The efficacy of a new program (The Penn Prevention Program) to prevent depressive symptoms and alleviate associated deficits in academic achievement, peer relations, self-esteem, and behavior is reported in 69 'at risk' 10-13 year-old children evaluated in the Department of Psychology, University of Pennsylvania, Philadelphia, PA. Using cognitive-behavioral techniques proactively to teach coping strategies, children at risk of developing depression, based on level of symptoms and perception of parental conflict, showed significant reductions in depressive symptoms and improved classroom behavior. Benefits were sustained at 6-month follow-up, especially in children with higher risk factors, as compared to control groups. (Jaycox LH et al. Prevention of depressive symptoms in school children. Behav Res Ther Nov 1994;32:801-816). (Respond: Dr Lisa H Jaycox, Department of Psychology, University of Pennsylvania, 3815 Walnut Street, Philadelphia, PA 19104).

COMMENT. The authors hypothesize that children at risk for depression use skills learned in the Penn Prevention Program to deal with conflict and avoid depressive symptoms precipitated by interpersonal problems. This type of program could be important in children with ADDH and learning problems who are at increased risk of developing depressive symptoms associated with poor self esteem and academic failure.

\section{HEAD INJURY}

\section{POST-HEAD INJURY FUNCTIONAL DEFICITS}

The functional outcome in 95 children (aged 5 to 15 ) at 1 year after hospitalization for head injury was evaluated at the Johns Hopkins University, School of Hygiene and Public Health, Baltimore, MD. More than half of all injuries were motor vehicle related: $21 \%$ pedestrians, $18 \%$ passengers, and $17 \%$ bicycles. Lower extremity injuries were sustained in 20. Severity of head injury was determined using the Abbreviated Injury Scale (AIS 2-5) and the Glasgow Coma Scale (GCS 3-15). GCS were highly correlated with AIS severity. Chronic health problems pre-dating the injury were reported in 23\%; these were minor in $15 \%$, and major (mental retardation, seizures, lead poisoning) in $8 \%$. After controlling for head injury severity, poorer outcomes were associated with poverty, preinjury chronic health problems, and lower extremity injuries. At 1 year follow-up, $55 \%$ had one or more health problems: headaches in $32 \%$, limb or peripheral nerve disorders $(13 \%)$, weakness or ataxia $(7 \%)$, and vision, hearing, or speech disorder $(6 \%)$. The presence of functional limitations in physical activity (31\%) or self-care mobility (19\%) was associated especially with severe head injuries (AIS 5), but those with AIS 2-4 were not spared at least one limitation. Hyperactive behavior was directly correlated with head injury severity, and head-injured children had a greater 